\title{
Camera Pose Measurement from 2D-3D Correspondences of Three Z Shaped Lines
}

\author{
Chang Liu ${ }^{1,2,3,4 *}$, Feng Zhu ${ }^{1,4}$, Jinjun Ou ${ }^{1,4}$, Yong Yu ${ }^{5}$, Renbo Xia ${ }^{1,4}$ \\ ${ }^{1}$ Shenyang Institute of Automation, Chinese Academy of Sciences, Shenyang 110016, China \\ ${ }^{2}$ School of Information Science \& Engineering, Shenyang Ligong University, Shenyang 110159, China \\ ${ }^{3}$ Graduate School of the Chinese Academy of Sciences, Beijing 100039, China \\ ${ }^{4}$ Key Laboratory of Opto-Electronic Information Processing, CAS, Shenyang 110016, China \\ ${ }^{5}$ Broadcom Corporation, Andover, MA 01810, U.S.A.
}

*Corresponding author's Email: syliuch@sia.cn

\begin{abstract}
Pose measurement is an important problem in computer vision. This paper presents a method for finding the closed form solutions to camera pose measurement problem by using 2D-3D correspondences of three $\mathrm{Z}$ shaped lines. This problem is a special case of Perspective-three-Line (P3L) problem. By introducing two undetermined parameters, we translate the problem into solving a quadratic equation. We also prove the problem's solution number depends on the relative position between the camera's optical center and the three $\mathrm{Z}$ shaped lines. A unique solution exists when the optical center locates inside two right circular cones. The experiments show the method is efficient. The method provides a new way using line features and monocular vision to pose measurement and can be applied to many vision systems.
\end{abstract}

Keywords: monocular vision; pose measurement; Perspective-three-Line (P3L); line feature; closed form solution

\section{Introduction}

Computer vision based pose measurement has been used in many fields such as aviation, robot navigation, industry automatic assembling, etc. If only one camera is used in a vision system, it is called monocular vision. Given some features of a target object, the position and orientation of the three-dimensional object can be estimated from the correspondences of features on the image with only one perspective view [1]. There are a variety of features which can be used such as points, lines, planes, corners, etc. Previous researchers attached much importance to point features. One of the most famous problems is Perspective-nPoint $(\mathrm{PnP})$ problem presented in [2] which $\mathrm{n}$ control points and correspondences for pose estimation are used. Many researchers studied this problem and their results have been used in many engineering applications successfully [3].

In real world, straight line is another important feature for many target objects. Pose measurement from line correspondences can also be applied to monocular vision systems. Perspective-n-Line $(\mathrm{PnL})$ problem needs to solve the problem of how to calculate position and orientation parameters from 2D-3D correspondences of $n$ lines. When the number of line correspondences is three, it is a $\mathrm{P} 3 \mathrm{~L}$ problem. Dhome et al. [4] researched a general case of the P3L problem and deduced an eight-degree polynomial equation. The P3L problem's solutions are the roots of the polynomial equation. By their methods, we can obtain eight possible solutions at most. It needs further conditions to judge which one is the real solution. 
Chen [5] updated Dhome's method and pointed out that in some cases the maximum number of real solutions to the problem is eight. Liu et al. [6] pointed out $\mathrm{P} 3 \mathrm{~L}$ problem can be solved by nonlinear approach and presented an iterative algorithm.

Generally, P3L problem has multiple solutions which make it difficult to use in real applications and unique solution conditions have drawn much attention in recent studies. It is found that if three lines on the target object have special geometric relationship such as parallelity or orthogonality, the problem has further properties. Shi et al. [7] studied a case that three lines intersect at one point. This is also a case of corner correspondence. They proved the existence of unique solution if given mutual angles among three lines and another point correspondence. Ying et al. [8] studied a case of parallel lines correspondence and applied to distinguish crosswalks and staircases aiding for the partially sighted. Frank [9] and Wang et al. [10] studied a case that four lines form a parallelogram and obtained a unique solution, but their problems both belong to P4L. Qin et al. [11, 12] studied a case that three lines intersect at two points and proved the maximum solution number is four in this case. Liu et al. [13] studied a case that three lines are shaped like a door and proved if the camera's optical center does not locates on a special plane, the problem has a unique solution.

In this paper, we study another special case of P3L that three lines on the object are $\mathrm{Z}$ shaped, which means two of three lines are parallel and the third line intersects with them at two points. If the camera's optical center has a special position in 3D region, the problem has a unique solution. This result makes it possible to estimate the pose parameters of a camera from a single perspective view and 2D to 3D correspondences of three $\mathrm{Z}$ shaped lines in monocular vision systems.

\section{Problem statement}

\subsection{Camera model and parameters}

In this paper we adopt pin-hole model for a camera. Suppose the camera has been calibrated. The camera's intrinsic parameters include: the principal point $\left(u_{o}, v_{o}\right)$, the focal ratio $f_{u}$ and $f_{v}$. The camera coordinate system is defined as follow: the origin is the camera's optical center; the $X$ axis and axis are respectively parallel to the $X$ axis and axis of the image; the $Z$ axis is the camera's optical axis which is perpendicular to the image plane. The object coordinates $\left(x_{W}, y_{W}, z_{W}\right)$ and the camera coordinates $\left(x_{C}, y_{C}, z_{C}\right)$

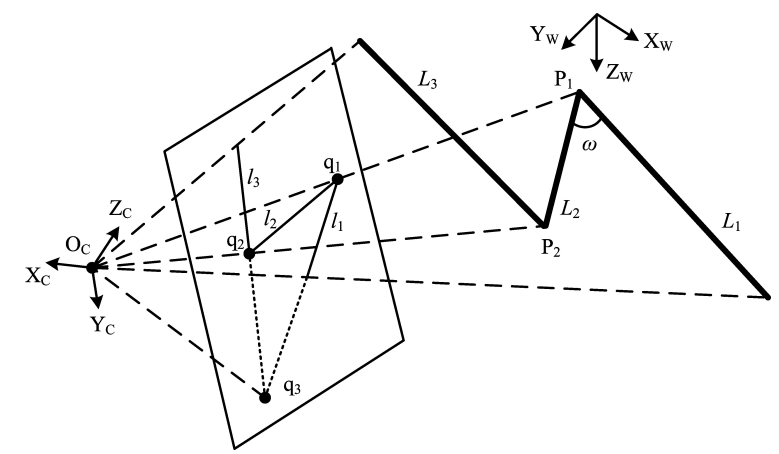

Figure $1 Z$ shaped $P 3 L$ problem formulation.

of a point satisfying

$$
\left(\begin{array}{l}
x_{C} \\
y_{C} \\
z_{C}
\end{array}\right)=R\left(\begin{array}{c}
x_{W} \\
y_{W} \\
z_{W}
\end{array}\right)+T,
$$

In equations 1 ,

$$
R=\left(\begin{array}{lll}
r_{11} & r_{12} & r_{13} \\
r_{21} & r_{22} & r_{23} \\
r_{31} & r_{32} & r_{33}
\end{array}\right)
$$

$R$ is the rotation matrix and $T=\left(T_{X}, T_{Y}, T_{Z}\right)^{T}$ is the translation vector.

Rotation matrix $R$ can be expressed by three rotation angles around three axes. In this paper let the angle around $X$ axis be $\psi$; the angle around $Y$ axis be $\phi$; the angle around $Z$ axis be $\theta$. The rotation order is $X, Y$, $Z$. We have

$$
\left\{\begin{array}{l}
r_{11}=\cos \theta \cos \phi \\
r_{12}=\sin \theta \cos \phi \\
r_{13}=-\sin \theta \\
r_{21}=\cos \theta \sin \phi \sin \psi-\sin \theta \cos \psi \\
r_{22}=\sin \theta \sin \phi \sin \psi+\cos \theta \cos \psi \\
r_{23}=\cos \phi \sin \psi \\
r_{31}=\cos \theta \sin \phi \cos \psi+\sin \theta \sin \psi \\
r_{32}=\sin \theta \sin \phi \cos \psi-\cos \theta \sin \psi \\
r_{33}=\cos \phi \cos \psi
\end{array}\right.
$$

From (3), it is not difficult to parse three rotation angles from a given rotation matrix $R$.

\subsection{Z shaped P3L problem formulation}

Figure.1 depicts the $Z$ shaped P3L problem. We have several requirements as follows:

1) Three lines $L_{1}, L_{2}$ and $L_{3}$ are coplanar and placed as Z shaped. $L_{1}$ and $L_{3}$ are rays and parallel to each other; $L_{2}$ intersects with $L_{1}$ and $L_{3}$ at $P_{1}$ and $P_{2}$; the angle between $L_{1}$ and $L_{2}$ is $\omega$. Because [13] has studied the case that $L_{2}$ is perpendicular to $L_{1}$ and $L_{3}$, here we 
only discuss the case $0<\omega<\pi / 2 . l_{1}, l_{2}$ and $l_{3}$ on the image plane correspond to lines $L_{1}, L_{2}$ and $L_{3} . q_{1}$ and $q_{2}$ on the image plane correspond to the intersection points $P_{1}$ and $P_{2}$.

2) The distance between $P_{1}$ and $P_{2}$ is $d(d>0)$.

3) In the camera coordinate system, the straight line equations of $l_{1}, l_{2}$ and $l_{3}$ on the image plane are given: $a_{i} x+b_{i} y+c_{i}=0(i=1,2,3)$. Here we only study the nonsingular case that the images of three lines are also three lines, they are not coincident or do not degenerate to points.

4) The line equations of $L_{1}, L_{2}$ and $L_{3}$ in the object coordinate system are given. Let $\vec{V}$ denotes a vector or the direction of a ray $V$. Here we suppose the object coordinate system is defined as: the origin is $P_{1}$; the $X$ axis is $L_{1}$ and its direction is $\overrightarrow{L_{1}}$; the $Y$ axis is on the plane which contains $L_{1}$ and $L_{2}$, perpendicular to $L_{1}$ and directs to $L_{3}$; the $Z$ axis is determined by the $X$ and $Y$ axes according to the right-hand rule.

5) All intrinsic parameters of the camera are given. The question is to find out the transformation ( $R$ and $T$ in (1)) between the object and the camera coordinates. The problem is equivalent to finding the coordinates description of three lines $L_{1}, L_{2}$ and $L_{3}$ in the camera coordinate system from their images.

Qin et al. [12] studied a similar problem and presented a method to obtain the problem's closed form solutions. They also pointed out that the problem has two different solutions. In [12], and $L_{1}$ and $L_{3}$ are supposed to be straight lines, while in this paper we suppose they are rays. Under this assumption, we find out the conditions that unique solution exists. Unique solution is important for pose determination in computer vision systems because we need not distinguish the real solutions from many possible ones.

\section{Closed form solutions to the problem}

\subsection{Direction of the $X$ axis of the object coordi- nate system}

If the camera's image plane is not parallel to $L_{1}$ and $L_{3}, l_{1}$ is not parallel to $l_{3}$. Suppose the intersection point of $l_{1}$ and $l_{3}$ is $q_{3} . q_{3}$ is the vanishing point of $L_{1}$ and $L_{3}$. Regarding the vanishing point, we have the following well-known lemma [14].

Lemma 1 : The line through the camera optical center and the vanishing point is parallel to the lines in the scene which have given rise to the vanishing point.

From Lemma 1 we have the ray $\overrightarrow{O_{C} q_{3}}$ which is parallel to $L_{1}$. Because $L_{1}$ is a ray, its image $l_{1}$ is also a ray. We can use the following method to obtain its direction: If the vanishing point $q_{3}$ is on $\overrightarrow{l_{1}}$, the direction of $L_{1}$ is $\overrightarrow{O_{C} q_{3}}$; if $q_{3}$ is on the oppositely elongated line of $\overrightarrow{l_{1}}$, the direction of $L_{1}$ is $\overrightarrow{q_{3} O_{C}}$.

If the camera's image plane is parallel to $L_{1}$ and $L_{3}$, then $l_{1} / / l_{3}$. The $X$ axis of the object coordinate system has a same direction with $\overrightarrow{l_{1}}$. Let $q_{3}$ be an arbitrary point on $\overrightarrow{l_{1}}$, then $\overrightarrow{q_{1} q_{3}}$ is the direction of the $X$ axis.

\subsection{Method for closed form solutions}

Let $f$ be the focal length of the camera. If the camera's image plane is not parallel to $L_{1}$ and $L_{3}$, the three intersection points are $q_{1}\left(x_{1}, y_{1}, f\right), q_{2}\left(x_{2}, y_{2}, f\right)$, $q_{3}\left(x_{3}, y_{3}, f\right)$, where

$$
\left\{\begin{array}{ll}
x_{1}=\frac{b_{1} c_{2}-b_{2} c_{1}}{a_{1} b_{2}-a_{2} b_{1}} & y_{1}=\frac{a_{2} c_{1}-a_{1} c_{2}}{a_{1} b_{2}-a_{2} b_{1}} \\
x_{2}=\frac{b_{2} c_{3}-b_{3} c_{2}}{a_{2} b_{3}-a_{3} b_{2}} & y_{2}=\frac{a_{3} c_{2}-a_{2} c_{3}}{a_{2} b_{3}-a_{3} b_{2}} \\
x_{3}=\frac{b_{1} c_{3}-b_{3} c_{1}}{a_{1} b_{3}-a_{3} b_{1}} & y_{3}=\frac{a_{3} c_{1}-a_{1} c_{3}}{a_{1} b_{3}-a_{3} b_{1}}
\end{array} .\right.
$$

Using the method in section 3.1, the direction of the $X$ axis of the object coordinate system is

$$
\overrightarrow{L_{1}}=\left(x_{3}, y_{3}, f\right)
$$

or

$$
\overrightarrow{L_{1}}=\left(-x_{3},-y_{3},-f\right) \text {. }
$$

If the camera's image plane is parallel to $L_{1}$ and $L_{3}$, suppose $\left(x_{0}, y_{0}\right)$ is an arbitrary point on the ray $l_{1}$, and let $x_{3}=x_{0}-x_{1}, y_{3}=y_{0}-y_{1}$, the direction of $X$ axis is

$$
\overrightarrow{L_{1}}=\left(x_{3}, y_{3}, 0\right) \text {. }
$$

To find solutions to the above problem, here we introduce two undetermined parameters. In the camera coordinate system, suppose $\overrightarrow{O_{C} P_{i}}=k \cdot \overrightarrow{O_{C} q_{i}}(i=1,2)$ where $k_{i}$ is the undetermined parameter whose geometric meaning is the ratio between the distance from $P_{i}$ to $O_{C}$ and the distance from $q_{i}$ to $O_{C}$. The direction of $L_{2}$ can be expressed as

$$
\begin{aligned}
\overrightarrow{L_{2}} & =\overrightarrow{P_{1} P_{2}}=k_{2} \cdot \overrightarrow{O_{C} q_{2}}-k_{1} \cdot \overrightarrow{O_{C} q_{1}} \\
& =\left(k_{2} x_{2}-k_{1} x_{1}, k_{2} y_{2}-k-1 y_{1},\left(k_{2}-k_{1}\right) f\right) .
\end{aligned}
$$

The angle between $L_{1}$ and $L_{2}$ is $\omega$, then

$$
\begin{aligned}
\cos \omega & =\frac{\left\|\overrightarrow{L_{1}} \cdot \overrightarrow{L_{2}}\right\|}{\left\|\overrightarrow{L_{1}}\right\|{ }^{1 / 2}\left\|\overrightarrow{L_{2}}\right\|^{1 / 2}} \\
& =\frac{\left\|\overrightarrow{O_{C} q_{3}} \cdot \overrightarrow{P_{1} P_{2}}\right\|}{\left\|\overrightarrow{O_{C} q_{3}}\right\|^{1 / 2}\left\|\overrightarrow{P_{1} P_{2}}\right\|^{1 / 2}} .
\end{aligned}
$$

The distance between $P_{1}$ and $P_{2}$ is $d$, then

$$
\sqrt{\left(k_{2} x_{2}-k_{1} x_{1}\right)^{2}+\left(k_{2} y_{2}-k_{1} y_{1}\right)^{2}+f^{2}\left(k_{2}-k_{1}\right)^{2}}=d .
$$


If the direction of $L_{1}$ is (5), from (9) and (10) we have

$$
\begin{aligned}
\left(x_{3} x_{2}+y_{3} y_{2}+f^{2}\right) k_{2}= & \left(x_{3} x_{1}+y_{3} y_{1}+f^{2}\right) k_{1} \\
& +d \cos \omega \sqrt{x_{3}^{2}+y_{3}^{2}+f^{2}} .
\end{aligned}
$$

There are following two possible cases.

(a) If $x_{3} x_{2}+y_{3} y_{2}+f^{2} \neq 0$, let

$$
\left\{\begin{array}{l}
s=\frac{x_{3} x_{1}+y_{3} y_{1}+f^{2}}{x_{3} x_{2}+y_{3} y_{2}+f^{2}} \\
r=\frac{d \cos \omega \sqrt{x_{3}^{2}+y_{3}^{2}+f^{2}}}{x_{3} x_{2}+y_{3} y_{2}+f^{2}}
\end{array} .\right.
$$

then (11) is rewritten into

$$
k_{2}=s k_{1}+r
$$

(13) depicts the linear relationship between $k_{2}$ and $k_{1}$. Put (13) into (10), we have

$$
\begin{array}{r}
\left(\left(s k_{1}+r\right) x_{2}-k_{1} x_{1}\right)^{2}+\left(\left(s k_{1}+r\right) y_{2}-k_{1} y_{1}\right)^{2} \\
+f^{2}\left(\left(s k_{1}+r\right)-k_{1}\right)^{2}=d^{2}
\end{array}
$$

Let

$\left\{\begin{array}{l}a=\left(s x_{2}-x_{1}\right)^{2}+\left(s y_{2}-y_{1}\right)^{2}+(s-1)^{2} f^{2} \\ b=2 r\left[\left(s x_{2}-x_{1}\right) x_{2}+\left(s y_{2}-y_{1}\right) y_{2}+(s-1)^{2} f^{2}\right] \\ c=r^{2}\left(x_{2}^{2}+y_{2}^{2}+f^{2}\right)-d^{2}\end{array}\right.$,

(14) can be rewrritten as

$$
a k_{1}^{2}+b k_{1}+c=0 .
$$

Because $q_{1}$ and $q_{2}$ are not coincident, we have $\left(x_{1}, y_{1}\right) \neq$ $\left(x_{2},\right)$, then $a>0$. (16) has two solutions

$$
k_{1}=\frac{-b \pm \sqrt{b^{2}-4 a c}}{2 a} .
$$

From (13), we can obtain two solutions of $k_{2}$ according to $k_{1}$. From the geometric meaning of $k_{1}$ and $k_{2}$, they must satisfy

$$
k_{1}>0 \text { and } k_{2}>0 \text {. }
$$

From (18), we can judge which one is the real solution to the problem.

(b) If $x_{3} x_{2}+y_{3} y_{1}+f^{2}=0$, (11) is rewritten into

$$
\begin{aligned}
& \left(x_{3} x_{1}+y_{3} y_{1}+f^{2}\right) k_{1} \\
& \quad+d \cos \omega \sqrt{x_{3}^{2}+y_{3}^{2}+f^{2}}=0 .
\end{aligned}
$$

From $d>0, \omega \neq \pi / 2$, and $\left\|\overrightarrow{O_{C} q_{3}}\right\| \neq 0$, it must have $x_{3} x_{1}+y_{3} y_{1}+f^{2} \neq 0$. Then we have

$$
k_{1}=-\frac{d \cos \omega \sqrt{x_{3}^{2}+y_{3}^{2}+f^{2}}}{x_{3} x_{1}+y_{3} y_{1}+f^{2}} .
$$

From (10), we have

$$
\begin{array}{r}
\left(x_{2}^{2}+y_{2}^{2}+f^{2}\right) k_{2}^{2}-2 k\left(x_{1} x_{2}+y_{1} y_{2}+f^{2}\right) k_{2} \\
+k_{1}^{2}\left(x_{1}^{2}+y_{1}^{2}+f^{2}\right)-d^{2}=0
\end{array}
$$

This is a quadratic equation with respect to $k_{2}$. let

$$
\left\{\begin{array}{l}
a^{\prime}=x_{2}^{2}+y_{2}^{2}+f^{2} \\
b^{\prime}=2 k\left(x_{1} x_{2}+y_{1} y_{2}+f^{2}\right) \\
c^{\prime}=k_{1}^{2}\left(x_{1}^{2}+y_{1}^{2}+f^{2}\right)-d^{2}
\end{array},\right.
$$

We can obtain two solutions of (21) as

$$
k_{2}=\frac{-b^{\prime} \pm \sqrt{b^{\prime 2}-4 a^{\prime} c^{\prime}}}{2 a^{\prime}} .
$$

Similarly, if the direction of $L_{1}$ is (6), from (9) and (10), we have

$$
\begin{aligned}
\left(x_{3} x_{2}+y_{3} y_{2}+f^{2}\right) k_{2}^{2} & =\left(x_{3} x_{1}+y_{3} y_{1}+f^{2}\right) k_{1} \\
- & d \cos \omega \sqrt{x_{3}^{2}+y_{3}^{2}+f^{2}} .
\end{aligned}
$$

If the direction of $L_{1}$ is (7), from (9) and (10), we have

$$
\begin{array}{r}
\left(x_{3} x_{2}+y_{3} y_{2}\right) k_{2}^{2}=\left(x_{3} x_{1}+y_{3} y_{1}\right) k_{1} \\
+d \cos \omega \sqrt{x_{3}^{2}+y_{3}^{2}} .
\end{array}
$$

We can obtain similar results in both cases. The above deductions justify the existence of two possible solutions at most. From criteria (18), we can distinguish the real solution to the problem.

Using the above method, we can obtain the coordinates of in the camera coordinate system, that is $\left(k_{1} x_{1}, k_{1} y_{1}, k_{1} f\right)$. As $P_{1}$ is the origin of the object coordinate system, its object coordinates are $(0,0,0)$. Then the translation vector is

$$
T=\left(T_{X}, T_{Y}, T_{Z}\right)^{T}=\left(k_{1} x_{1}, k_{1} y_{1}, k_{1} f\right)^{T} .
$$

We can also obtain the direction of $\overrightarrow{L_{2}}$ from (8) after we get the camera coordinates of $P_{1}$ and $P_{2}$. It is not difficult to obtain the directions of the three axes of the object coordinate system and the rotation matrix $R$. From the above method, the problem's closed form solutions are resolved. 


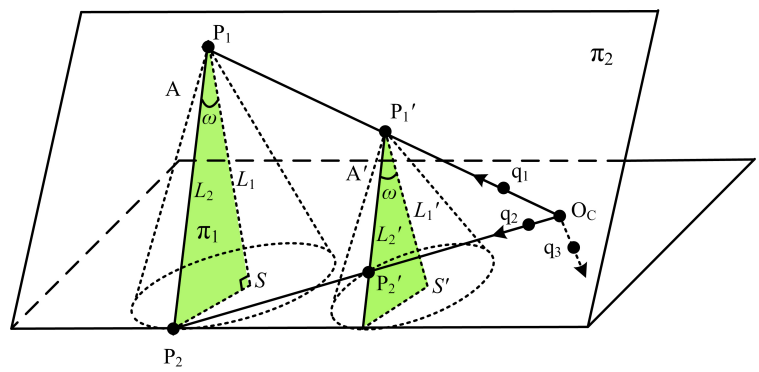

Figure 2 Illustration of Theorem 1.

\section{Problem's solution number}

Unique solution conditions are important for P3L in real vision applications. Regarding the problem solution's properties, we have the following theorems.

Theorem 1: Let the plane which contains $L_{1}, L_{2}$ and $L_{3}$ be $\pi_{1}$; the plane which is perpendicular to $\pi_{1}$ and passes $L_{2}$ be $\pi_{2}$. If the camera's optical center locates on $\pi_{2}$, the problem has a unique solution.

\subsection{Z shaped P3L problem formulation}

Proof: As shown in Figure.2, assuming that $L_{1}, L_{2}$ and $L_{3}\left(L_{3}\right.$ is not shown in Figure. 2$)$ are the real solution lines of the problem, whose intersection points are $P_{1}$ and $P_{2}$, from the given conditions, the angle between $L_{1}$ and $L_{2}$ is $\omega$, and $\left|P_{1} P_{2}\right|=d$. Make a right circular cone $A$; let the vertex of $A$ be $P_{1}$, the axis of $A$ be $L_{1}$, and the vertex angle of $A$ be $2 \omega, L_{2}$ must locate on the surface of the cone $A$. From the definition of $\pi_{2}$, the cone $A$ is tangent with $\pi_{2}$ and the tangent line between $A$ and $\pi_{2}$ is $L_{2}$.

Similarly, assuming that $L_{1}^{\prime}, L_{2}^{\prime}$ and $L_{3}^{\prime}$ ( $L_{3}^{\prime}$ is not shown in Figure.2) are also solution lines of the problem. Their intersection points are $P_{1}^{\prime}$ and $P_{2}^{\prime}$. Because we have obtained the direction of $L_{1}$ in section 3.1, it must have $L_{1} / / L_{1}^{\prime}$. From the given conditions, $P_{1}^{\prime}$ is on the ray $\overrightarrow{O_{C} P_{1}} ; P_{2}^{\prime}$ is on the ray $\overrightarrow{O_{C} P_{2}} ;\left|P_{1}^{\prime} P_{2}^{\prime}\right|=d$; the angle between $L_{1}^{\prime}$ and $L_{2}^{\prime}$ is also $\omega$. Make another right circular cone $A^{\prime}$; let the vertex of $A^{\prime}$ be $P_{1}^{\prime}$, the axis of $A^{\prime}$ be $L_{1}^{\prime}$, and the vertex angle of $A^{\prime}$ be $2 \omega$. Then $L_{2}^{\prime}$ must locate on the surface of the cone $A^{\prime}$; the cone $A^{\prime}$ must be tangent with $\pi_{2}$; and the tangent line between $A^{\prime}$ and $\pi_{2}$ is $L_{2}^{\prime}$.

Regarding two triangles $O_{C} P_{1} P_{2}$ and $O_{C} P_{1}^{\prime} P_{2}^{\prime}$, we have $P_{1} P_{2} / / P_{1}^{\prime} P_{2}^{\prime}$. Because the real solution must satisfy $\left|P_{1} P_{2}\right|=\left|P_{1}^{\prime} P_{2}^{\prime}\right|=d$, the two triangles $O_{C} P_{1} P_{2}$ and $O_{C} P_{1}^{\prime} P_{2}^{\prime}$ must be coincident. Then the two solutions are coincident. The problem has a unique solution. This proves Theorem 1.

Because a plane is not a 3D space, Theorem 1 only

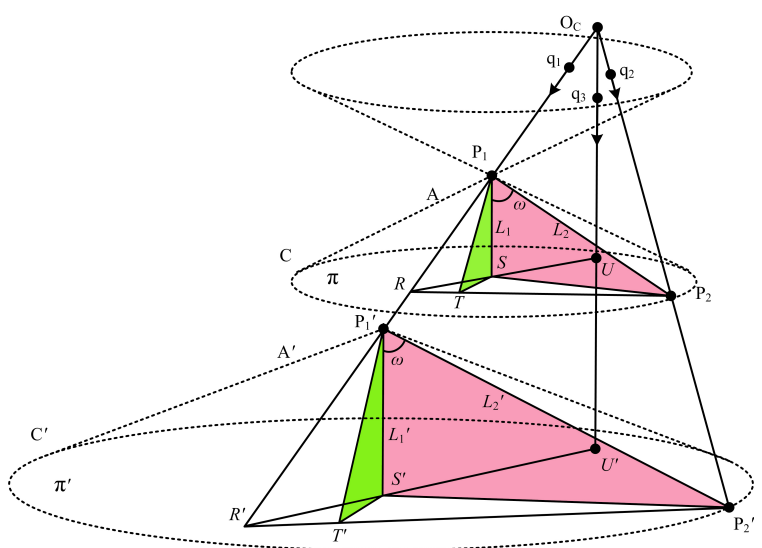

Figure 3 Illustration of Theorem 2.

has theoretical meaning. It shows $\pi_{2}$ is an important reference plane for the problem.

Theorem 2: Let the angle between $L_{1}$ and $O_{C} q_{1}$ be an acute angle. If the angle is less than $\omega$, the problem has a unique solution.

Proof: As shown in Figure.3, assuming that $L_{1}, L_{2}$ and $L_{3}$ ( $L_{3}$ is not shown in Figure. 3) are the real solution lines of the problem whose intersection points are $P_{1}$ and $P_{2}$, from the given conditions, $P_{1}$ is on the ray $\overrightarrow{O_{C} q_{1}} ; P_{2}$ is on the ray $\overrightarrow{O_{C} q_{2}} ;\left|P_{1} P_{2}\right|=d$ and the angle of $L_{1}$ and $L_{2}$ is $\omega$. Make a right circular cone $A$, let the vertex of $A$ be $P_{1}$, the axis of $A$ be $L_{1}$; the vertex angle of $A$ be $2 \omega, L_{2}$ must locate on the surface of the cone $A$. Because the angle of $L_{1}$ and $\overrightarrow{O_{C} P_{1}}$ is less than $\omega$, there is only one intersection point of $\overrightarrow{O_{C} q_{2}}$ and the surface of the cone $A$, let it be $P_{2}$. Make a plane $\pi$, let it be perpendicular to $L_{1}$ and pass $P_{2}$. Suppose the cone $A$ intersects with $\pi$ at circle $C$, let the intersection point of $L_{1}$ and $\pi$ be $S$, the intersection point of the ray $\overrightarrow{O_{C} q_{3}}$ and $\pi$ be $U$, the intersection point of $\overrightarrow{O_{C} q_{1}}$ and $\pi$ be $R$. Obviously, the three points $R, S$ and $U$ are collinear.

Similarly, assuming that $L_{1}^{\prime}, L_{2}^{\prime}$ and $L_{3}^{\prime}$ is not shown in Figure.3) are also solution lines of the problem. Their intersection points are $P_{1}^{\prime}$ and $P_{2}^{\prime}$. From the given conditions we have $P_{1}^{\prime}$ on $\overrightarrow{O_{C} q_{1}}, P_{2}^{\prime}$ on $\overrightarrow{O_{C} q_{2}}$; the angle between $L_{1}^{\prime}$ and $L_{2}^{\prime}$ is also $\omega ;\left|P_{1}^{\prime} P_{2}^{\prime}\right|=d ; L_{1} / / L_{1}^{\prime} / / \overrightarrow{O_{C} q_{3}}$. Make another right circular cone $A^{\prime}$; let the vertex of $A^{\prime}$ be $P_{1}^{\prime}$, the axis of $A^{\prime}$ be $L_{1}^{\prime}$, and the vertex angle of $A^{\prime}$ be $2 \omega, L_{2}^{\prime}$ must locate on the surface of the cone $A^{\prime}$. Because the angle of $L_{1}^{\prime}$ and $O_{C} P_{1}^{\prime}$ is less than $\omega$, there is also only one intersection point of $\overrightarrow{O_{C} q_{2}}$ and the surface of the cone $A^{\prime}$, let it be $P_{2}^{\prime}$. Make a plane $\pi^{\prime}$, let it be perpendicular to $L_{1}^{\prime}$ and pass $P_{2}^{\prime}$. Suppose the cone $A^{\prime}$ intersects with $\pi^{\prime}$ at circle $C^{\prime}$. Let the intersection point of $L_{1}^{\prime}$ and $\pi^{\prime}$ is $S^{\prime}$; the intersection point 


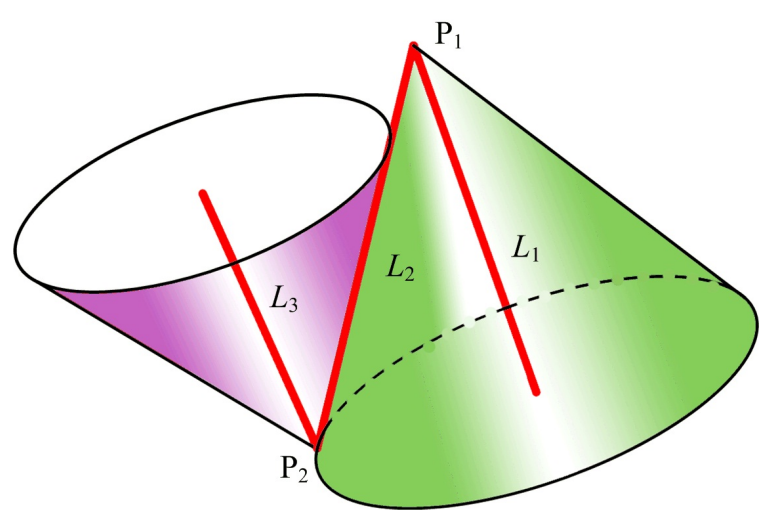

Figure 4 The unique solution region to the problem.

of $\overrightarrow{O_{C} q_{3}}$ and $\pi^{\prime}$ is $U^{\prime}$; the intersection point of $\overrightarrow{O_{C} q_{1}}$ and $\pi^{\prime}$ is $R^{\prime}$. It is clear that three points $R^{\prime}, S^{\prime}$ and $U^{\prime}$ are collinear.

The intersection line of plane $O_{C} q_{1} q_{2}$ and $\pi$ is $R P_{2}$, the intersection line of plane $O_{C} q_{1} q_{2}$ and $\pi^{\prime}$ is $R^{\prime} P_{2}^{\prime}$, and $\pi^{\prime} / / \pi$, then we have $R P_{2} / / R^{\prime} P_{2}^{\prime}$.

Make $P_{1} T \perp P_{2} R$ at $T, P_{1}^{\prime} T^{\prime} \perp P_{2}^{\prime} R^{\prime}$ at $T^{\prime}$. From $R P_{2} / / R^{\prime} P_{2}^{\prime}$ we have $P_{1} T / / P_{1}^{\prime} T^{\prime}$. From $P_{1} S / / O_{C} q_{3} / / P_{1}^{\prime} S^{\prime}$ we have $\angle T P_{1} S=\angle T^{\prime} P_{1}^{\prime} S^{\prime}$. From $P_{1} S \perp S T$ and $P_{1}^{\prime} S^{\prime} \perp$ $S^{\prime} T^{\prime}$ we have triangle $P_{1} S T$ is similar to triangle $P_{1}^{\prime} S^{\prime} T^{\prime}$, then we have

$$
\frac{P_{1} T}{P_{1}^{\prime} T^{\prime}}=\frac{P_{1} S}{P_{1}^{\prime} S^{\prime}}
$$

where $P_{2}$ and $P_{2}^{\prime}$ are on the surface of $A$ and $A^{\prime}$ respectively, and the vertex angle of $A$ and $A^{\prime}$ are both $2 \omega$, we have $\angle P_{2} P_{1} S=\angle P_{2}^{\prime} P_{1}^{\prime} S^{\prime}$. From $P_{1} S \perp S P_{2}$ and $P_{1}^{\prime} S^{\prime} \perp S^{\prime} P_{2}^{\prime}$, we have triangle $P_{1} S P_{2}$ is similar to triangle $P_{1}^{\prime} S^{\prime} P_{2}^{\prime}$, then

$$
\frac{P_{1} P_{2}}{P_{1}^{\prime} P_{2}^{\prime}}=\frac{P_{1} S}{P_{1}^{\prime} S^{\prime}}=\frac{P_{1} T}{P_{1}^{\prime} T^{\prime}}
$$

where $P_{1} T P_{2}$ and $P_{1}^{\prime} T^{\prime} P_{2}^{\prime}$ are both right triangles, they are similar to each other. Then $\angle T P_{2} P_{1}=\angle T^{\prime} P_{2}^{\prime} P_{1}^{\prime}$. From $R P_{2} / / R^{\prime} P_{2}^{\prime}$, and as $R P_{2}, R^{\prime} P_{2}^{\prime}, P_{1} P_{2}, P_{1}^{\prime} P_{2}^{\prime}$ four lines are coplanar, we have $P_{1} P_{2} / / P_{1}^{\prime} P_{2}^{\prime}$.

Regarding two triangles $O_{C} P_{1} P_{2}$ and $O_{C} P_{1}^{\prime} P_{2}^{\prime}$, because $P_{1} P_{2} / / P_{1}^{\prime} P_{2}^{\prime}$ and $\left|P_{1} P_{2}\right|=\left|P_{1}^{\prime} P_{2}^{\prime}\right|=d$, we conclude they must be coincident. Then the two solutions of the problem are same. The problem has a unique solution. This proves Theorem 2 .

Theorem 3: Let the angle between $L_{1}$ and $O_{C} q_{2}$ be an acute angle. If the angle is less than $\omega$, the problem has a unique solution. The proof of Theorem 3 is similar to that of Theorem 2 .

Theorem 2 and Theorem 3 define a 3D region constituted of four right circular cones, whose vertexes

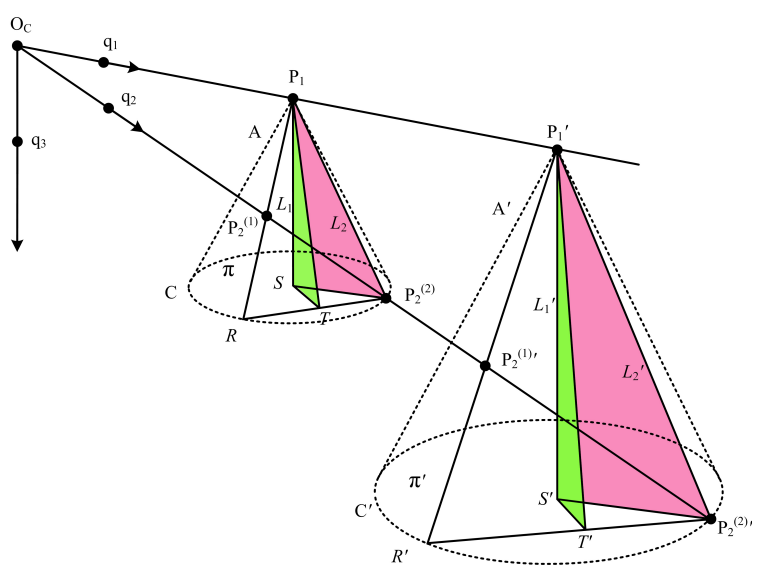

Figure 5 Illustration of Theorem 4.

are $P_{1}$ and $P_{2}$, vertex angles are $2 \omega$ and axes are $L_{1}$ and $L_{3}$ respectively. Regarding these four cones, it is not difficult to prove that two of them are included by the other two cones. As shown in Figure.4, if the optical center locates inside the two cones, the problem has a unique solution.

Theorem 4: If the optical center does not locate into the region described in Theorem 1, Theorem 2 or Theorem 3, the problem has two solutions.

Proof: As shown in Figure.5, make two right circular cone $A$ and $A^{\prime}$, let their vertexes be $P_{1}$ and $P_{1}^{\prime}$ which are two points on the ray $\overrightarrow{O_{C} q_{1}}$; their axes be parallel to $\overrightarrow{\mathrm{O}_{C} q_{3}}$ which we obtain from section 3.1 ; their vertex angles be both $2 \omega$. If the optical center does not locate in the region described before, there are two intersection points of $\overrightarrow{O_{C} q_{2}}$ and each cone. Let the intersection points of $\overrightarrow{O_{C} q_{2}}$ and $A$ be $P_{2}^{(1)}$ and $P_{2}^{(2)}$; the intersection points of $\overrightarrow{O_{C} q_{2}}$ and $A^{\prime}$ be $P_{2}^{(1)^{\prime}}$ and $P_{2}^{(2)^{\prime}}$. Similar to the proof of Theorem 2, triangle $P_{1} S T$ is similar to $P_{1}^{\prime} S^{\prime} T^{\prime} ; P_{1} S P_{2}^{(2)}$ is similar to $P_{1}^{\prime} S^{\prime} P_{2}^{(2)^{\prime}}$, then

$$
\frac{P_{1} P_{2}^{(2)}}{P_{1}^{\prime} P_{2}^{(2)^{\prime}}}=\frac{P_{1} S}{P_{1}^{\prime} S^{\prime}}=\frac{P_{1} T}{P_{1}^{\prime} T^{\prime}} .
$$

Then two right triangles $P_{1} T P_{2}^{(2)}$ and $P_{1}^{\prime} T^{\prime} P_{2}^{(2)^{\prime}}$ are similar to each other and $\angle T P_{2}^{(2)} P_{1}=\angle T^{\prime} P_{2}^{(2)^{\prime}} P_{1}^{\prime}$. From $R P_{2}^{(2)} / / R^{\prime} P_{2}^{(2)^{\prime}}$ and $R P_{2}^{(2)}, R^{\prime} P_{2}^{(2)^{\prime}}, P_{1} P_{2}^{(2)}, P_{1}^{\prime} P_{2}^{(2)^{\prime}}$ are coplanar, we have

$$
P_{1} P_{2}^{(2)} / / P_{1}^{\prime} P_{2}^{(2)^{\prime}} .
$$

Similarly, we have

$$
P_{1} P_{2}^{(1)} / / P_{1}^{\prime} P_{2}^{(1)^{\prime}} .
$$


From Equations (30) and (31), there are only two possible directions of $P_{1} P_{2}$ which may satisfy the problem's conditions on angle constrains. To find out the location of $L_{2}$, we need to move $P_{1}$ on the ray $\overrightarrow{O_{C} q_{1}}$ from its end point $O_{C}$, according to the direction $P_{1} P_{2}^{(1)}$ or $P_{1} P_{2}^{(2)}$, for the segment $P_{1} P_{2}$ satisfied $\left|P_{1} P_{2}\right|=d$. Obviously there is only one possible segment $P_{1} P_{2}$ on each direction satisfied the condition. Then the problem has two solutions. This proves Theorem 4.

The above four theorems show the problem's solution number depends on the relative position between the optical center and the three lines. For real applications, if we control the camera's optical center into the 3D region described in Figure.4, we can obtain the unique solution of the problem.

\section{Experimental results and analysis}

\subsection{Simulations}

Experiment 1: Select three $\mathrm{Z}$ shaped lines satisfying $\omega=45^{\circ}$ and $d=141.4 \mathrm{~mm}$. Suppose the image size is $512 \times 512$; the camera's focal ratio at two directions are both 2000; the principle point is $(256,256)$. Let the translation vector be $\left(T_{X}, T_{Y}, T_{Z}\right)=(0 \mathrm{~mm}, 0 \mathrm{~mm}$ $2000 \mathrm{~mm}$ ), and three rotation angles be $\psi=0^{\circ}, \phi=$ $50^{\circ}$, and $\theta=0^{\circ}$. Suppose there is no error on image and lines detection. The experimental results of pose parameters are shown in Table 1. For the first solution, $k_{1}>0$ and $k_{2}>0$, it is a correct solution. For the second solution, $k_{1}<0$, which does not satisfy (18), so it is not a reasonable solution. There is only one solution for the case. In this experiment, the angle $\angle q_{1} O_{C} q_{3}=40^{\circ}$, and $\angle q_{2} O_{C} q_{3}=38.3^{\circ}$. The camera's optical center locates inside the circular cones in Figure.4. This experiment verifies Theorem 2 and Theorem 3.

Table 1 Results of Experiment 1

\begin{tabular}{|c|c|c|c|c|c|c|c|c|}
\hline NO. & $k_{1}$ & $k_{2}$ & $T_{X}$ & $T_{Y}$ & $T_{Z}$ & $\psi$ & $\phi$ & $\theta$ \\
\hline 1 & 125 & 129.8 & 0 & 0 & 2000 & 0 & 50 & 0 \\
\hline 2 & -31.3 & -22.5 & - & - & - & - & - & - \\
\hline
\end{tabular}

Experiment 2: The experiment conditions are similar to that of Experiment 1. Let The translation vector is also $\left(T_{X}, T_{Y}, T_{Z}\right)=(0 \mathrm{~mm}, 0 \mathrm{~mm}, 2000 \mathrm{~mm})$ and three rotation angles be $\psi=0^{\circ}, \phi=40^{\circ}$, and $\theta=0^{\circ}$. The experimental results are shown in Table 2. Both solutions have $k_{1}>0$ and $k_{2}>0$, so they are both possible solutions. In this experiment, $\angle q_{1} O_{C} q_{3}=50^{\circ}$, and $\angle q_{2} O_{C} q_{3}=47.9^{\circ}$. The optical center is outside the circular cones in Figure.4. This experiment verifies

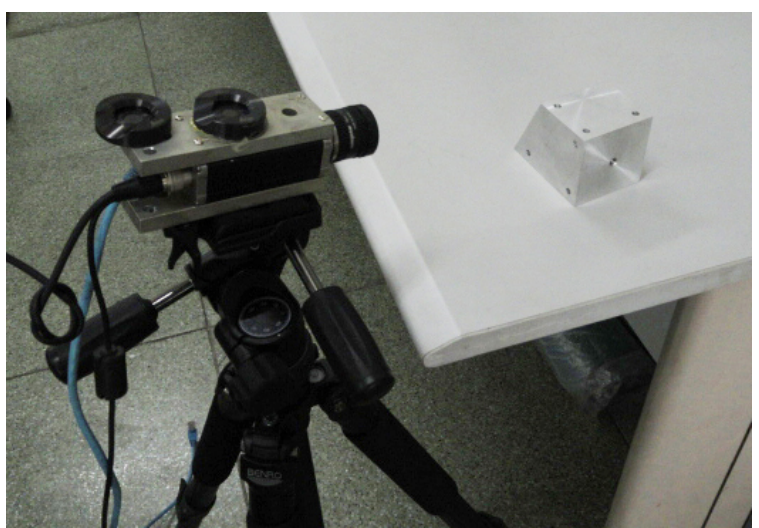

Figure 6 Experiment system of Experiment 3.

Theorem 4.

Table 2 Results of Experiment 2

\begin{tabular}{|c|c|c|c|c|c|c|c|c|}
\hline NO. & $k_{1}$ & $k_{2}$ & $T_{X}$ & $T_{Y}$ & $T_{Z}$ & $\psi$ & $\phi$ & $\theta$ \\
\hline 1 & 125 & 129 & 0 & 0 & 2000 & 0 & 40 & 0 \\
\hline 2 & 13.7 & 22.5 & 0 & 0 & 218.7 & 97.7 & 173.6 & 140.4 \\
\hline
\end{tabular}

\subsection{Real data}

Experiment 3: The experiment system is shown in Figure.6. We fix a camera on a tripod. The camera we used in the experiment is Pylon piA640-210gm produced by Basler. The image size is $640 \times 480$. A model with three $\mathrm{Z}$ shaped edge lines is put in front of the camera which is calibrated in advance. Here $\omega=65.43^{\circ}$ and $d=73.38 \mathrm{~mm}$. The pose parameters, which are expressed by rotation angles and translation vector, between the model and the camera are measured by a Leica AT901-LR absolute tracker in this experiment. The measured data is exact enough to be considered as reference standard in data verification. We take a photo of the model by the camera, while keeping the position and attitude between the model and the camera unchanged. Figure.7(a) is the photo of the model taken by the camera. We first detect the edge points of the image by Canny operator and then detect the straight lines on the image by Hough transform algorithm. Generally, there are many possible lines detected, here we select three as the $\mathrm{Z}$ shaped edge lines of the model by ourselves. Figure.7(b) is the edge image after Canny detection and we also mark the three lines selected for calculation in Figure.7(a). After we obtain the equations of three lines on the image, we calculate the rotation matrix and translation vector by the proposed method.

The results measured by the laser tracker and calculated from the image are listed in Table 3. From Table 


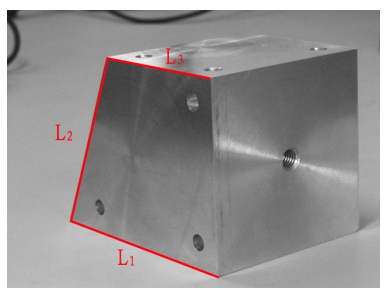

(a)

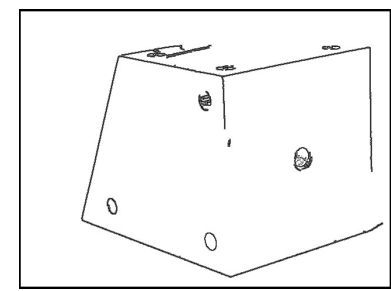

(b)
Figure 7 Images in Experiment 3.

3 , the two results are close to each other. The maximum error of the elements of the translation vector is less than $5 \%$, and the maximum error of the rotation angles is less than $2^{\circ}$.

Table 3 Results of Experiment 3

\begin{tabular}{|c|c|c|}
\hline & measured results & calculated results \\
\hline$\psi$ & $163.445^{\circ}$ & $165.299^{\circ}$ \\
\hline$\phi$ & $51.512^{\circ}$ & $51.972^{\circ}$ \\
\hline$\theta$ & $356.085^{\circ}$ & $357.302^{\circ}$ \\
\hline$T_{X}$ & $-45.612 \mathrm{~mm}$ & $-44.713 \mathrm{~mm}$ \\
\hline$T_{Y}$ & $35.594 \mathrm{~mm}$ & $33.982 \mathrm{~mm}$ \\
\hline$Y_{Z}$ & $508.295 \mathrm{~mm}$ & $489.577 \mathrm{~mm}$ \\
\hline
\end{tabular}

Experiment 4: The objective of this experiment is to measure the relative position between two cards (see Figure.8). Two cards K and Q are placed on a table and we take a photo with a camera calibrated in advance. The ruler-measured distance between two corner points of cards is $90.0 \mathrm{~mm}$. As shown in Figure.8, we set two object coordinate systems $\left(x_{1}, y_{1}, z_{1}\right)$ and $\left(x_{2}, y_{2}, z_{2}\right)$. Suppose that

$$
\left(\begin{array}{l}
x_{C} \\
y_{C} \\
z_{C}
\end{array}\right)=R_{1}\left(\begin{array}{l}
x_{1} \\
y_{1} \\
z_{1}
\end{array}\right)+T_{1} \text { and }\left(\begin{array}{l}
x_{C} \\
y_{C} \\
z_{C}
\end{array}\right)=R_{2}\left(\begin{array}{l}
x_{2} \\
y_{2} \\
z_{2}
\end{array}\right)+T_{2}
$$

then we have

$$
\begin{aligned}
\left(\begin{array}{l}
x_{2} \\
y_{2} \\
z_{2}
\end{array}\right) & =R_{2}^{-1}\left(\begin{array}{l}
x_{C} \\
y_{C} \\
z_{C}
\end{array}\right)-R_{2}^{-1} T_{2} \\
& =R_{2}^{-1} R_{1}\left(\begin{array}{l}
x_{1} \\
y_{1} \\
z_{1}
\end{array}\right)+R_{2}^{-1}\left(T_{1}-T_{2}\right)
\end{aligned}
$$

Using the frame lines of cards as control lines, we calculate the transformation between two coordinate systems. The results are

$$
\begin{aligned}
\left(\begin{array}{l}
x_{2} \\
y_{2} \\
z_{2}
\end{array}\right) & =\left(\begin{array}{ccc}
0.364 & 0.931 & 0.010 \\
0.931 & -.0364 & 0.007 \\
0.010 & 0.007 & -1.000
\end{array}\right)\left(\begin{array}{l}
x_{1} \\
y_{1} \\
z_{1}
\end{array}\right) \\
& +\left(\begin{array}{c}
-55.452 \\
71.826 \\
-6.226
\end{array}\right)
\end{aligned}
$$

After parsing from the rotation matrix, three rotation angles are $\psi=179.61^{\circ}, \phi=-0.59^{\circ}, \theta=68.66^{\circ}$. The

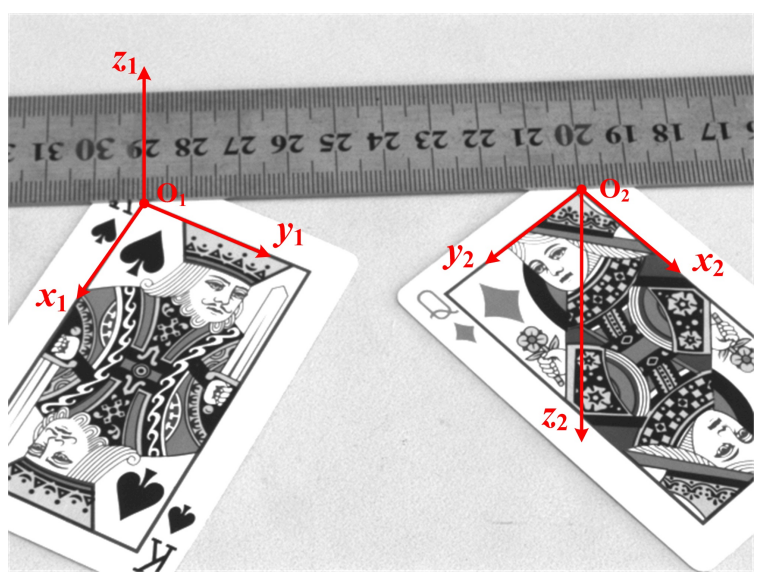

Figure 8 Image of Experiment 4.

distance between $O_{1}$ and $O_{2}$ is

$\sqrt{(-55.452)^{2}+71.286^{2}+(-6.226)^{2}}=90.954(\mathrm{~mm})$

This result approximates the real values. It shows the method in this paper is efficient.

\section{Conclusion}

By geometric deduction, we proved that the solution number of $Z$ shaped P3L problem depends on the relative position between the camera's optical center and the three $\mathrm{Z}$ shaped lines. The main conclusions include:

1) If optical center locates inside the $3 \mathrm{D}$ region of two right circular cones, as shown in Figure.4, whose vertex angles are both $2 \omega$, vertexes are $P_{1}$ and $P_{2}$, and axes are $L_{1}$ and $L_{3}$ respectively, the problem has a unique solution. The space inside two cones extends with the increase of vertex angle, which is advantageous to pose measurement.

2) If optical center locates on the specific plane described in Theorem 1, the problem also has a unique solution. But because a plane is not a $3 \mathrm{D}$ space, the plane is only an important reference plane.

3) If optical center locates on the plane which contains $L_{1}, L_{2}$ and $L_{3}$, the problem is degenerated because the images of three lines are coincident. We did not discuss this case in the paper.

4) If optical center has other locations, the problem has two solutions. It needs further criteria to distinguish which one is the real solution.

To summarize, it is possible to use three $\mathrm{Z}$ shaped lines to pose measurement between the camera and the target object. The problem's solutions can also be resolved by a closed form method presented in this paper. Its performance is more effective than iterative 
methods. Experiment results show the efficiency of the method. It provides us a new pose measurement method in computer vision systems.

\section{Acknowledgments}

This work is supported by the National Nature Science Foundation of China (No.60705011 and No.51005229).

\section{References}

[1] T. Huang, and A. Netravali, "Motion and Structure from Feature Correspondences: A Review", In: Proc. IEEE. Vol.82, No.2, pp.252-268, 1994.

[2] M. A. Fischler, and R. C. Bolles, "Random Sample Consensus: A Paradigm for Model Fitting with Apphcatlons to Image Analysis and Automated Cartography", In: Commun. ACM, vol.24, No.6, pp.381$395,1981$.

[3] Y. Wu, and Z. Hu, "PnP Problem Revisited", In: Journal of Mathematical Imaging and Vision, vol.24, pp.131-141, 2006.

[4] M. Dhome, M. Richetim, J. Laprseste, and G. Rives, "Determination of the Attitude of 3-D Objects from a Single Perspective View", In: IEEE Transactions on PAMI, Vol.11, No.12, pp.68-73, 1989.

[5] H. Chen, "Pose Determination from Line-to-Plane Correspondences: Existence Condition and ClosedForm Solutions", In: IEEE Transactions on PAMI, Vol.13, No.6, pp.530-541, 1991.

[6] Y. Liu, T. Huang, and O. Faugeras, "Determination of Camera Location from 2-D to 3-D Line and Point Correspondences", In: IEEE Transactions on PAMI, Vol.12, No.1, pp.28-37, 1990.

[7] F. Shi, X. Zhang, and Y. Liu, "A New Method of Camera Pose Estimation Using 2D-3D Corner Correspondence", In: Pattern Recognition letters, Vol.25, pp.1155-1163, 2004.

[8] X. Ying, and H. Zha, "Camera Pose Determination From a Single View of Parallel Lines", In: Proc. IEEE ICIP, pp.1056-1059, 2005.

[9] A. Frank, "Exterior Orientation using Coplanar Parallel Lines", In: Proc. of the 10th Scandinavian Conference on Image Analysis, Lappeenranta, pp.71-78, 1997.

[10] X. Wang, S. Pan, L. Qiu, W. Shen, and Z. Song, "Analytic algorithm of pose estimation based on two pairs of parallel lines", In: Chinese Journal of Scientific Instrument, Vol.29, No.3, pp.600-604, 2008.

[11] L. Qin, "Research on Pose Estimation Method and Robustness Analysis from Line Correspondences", Shenyang Institute of Automation, Chinese Academy of Sciences, Doctoral of Science thesis, 2007.
[12] L. Qin, Y. Hu, Y. Wei, Y. Zhou, and H. Wang, "A New Closed-Form Method for Pose Estimation from Three Z-like Lines", In: Proc. 7th World Congress on Intelligent Control and Automation, pp.4217-4221, 2008.

[13] C. Liu, F. Zhu, and J. Ou, "Door Shaped Three Lines Based P3L Problem's Closed-Form Solution". In: Transactions of Shenyang Ligong University, Vol.28, No.4, pp.11-14, 2009.

[14] P. Beardsley, and D. Murray, "Camera Calibration using Vanishing Points", In: Proc.BMVC, pp.146-425, 1992 